\title{
Electropulsing-Induced Microstructural Changes and Their Effects on Electrical Conductivity of Thin Films of an Al-doped $\mathrm{ZnO}$
}

\author{
Y. H. Zhu' ${ }^{1}$ C. M. Luk ${ }^{2}$ \\ Received: 3 January 2018/Revised: 4 April 2018/Published online: 17 October 2018 \\ (C) The Chinese Society for Metals and Springer-Verlag GmbH Germany, part of Springer Nature 2018
}

\begin{abstract}
Electropulsing-induced phase decompositions and microstructural changes in AZO-5 thin films were studied by X-ray diffraction, scanning electron microscopy, atomic force microscopy, Hall effect measurement and photoluminescence (PL) measurement techniques. It was found that the electropulsing induced phase decomposition started with spinodal decomposition, which was accompanied by discontinuous precipitation in the AZO-5 thin films. Both circular phase decompositions and the crystal orientation occurred. Inappropriate electropulsing might damage zones, which resulted in tremendous decrease in electrical conductivity. Circular changes in both the peak position and the width of the PL wavelength were observed in the EPT AZO-5 thin films. Formation of zones favored reducing the roughness of the thin film.
\end{abstract}

Keywords Electropulsing · Phase transformation - Electrical conductivity · Photoluminescence $\cdot$ Thin-films . AZO

\section{Introduction}

Transparent conductive oxide (TCO) thin films have received considerable attention due to their applications in various electronic and optoelectronic devices [1-4]. Aldoped zinc oxide (AZO) thin film is the most promising candidate because of its good electrical and optical properties, and low cost $[5,6]$. AZO thin films are of high chemical and physical stability, which makes it difficult to study structural evolution mechanism.

In the last decade, systematic studies of electropulsinginduced phase transformations and microstructural changes in various alloys, such as $\mathrm{Zn}-\mathrm{Al}$ [7-12], $\mathrm{Mg}-\mathrm{Al}$ [13-18], $\mathrm{Fe}-\mathrm{Si}$ alloys [19, 20], Cu based alloys and Ti based alloys [21-23] have been carried out. It was reported that plastic behavior might be much improved [7, 9, 10, 13, 14], and

Available online at http://link.springer.com/journal/40195

Y. H. Zhu

yaohuazhu@hotmail.com

1 Department of Industrial and Systems Engineering, The Hong Kong Polytechnic University, Hong Kong, China

2 Department of Applied Physics, The Hong Kong Polytechnic University, Hong Kong, China the phase decomposition and recrystallization were tremendously accelerated [7-20]. Circulations of both microstructural changes and the related mechanical property responses were quantitatively estimated [7-11, 16-19].

In addition, electropulsing-induced microstructural changes in the stable thin films of the $\mathrm{Zn}-\mathrm{Al}$ alloy and the functional semiconductors, such as Bi-Te and AZO-2, became focused research interests [24-26]. Previous studies showed that electropulsing was proved to be an effective and practical way to explore structural evolution of the thin films of the semiconductors with appropriate properties required for the electronic devices.

In the present work, electropulsing-induced phase decompositions and microstructural changes and their effects on some physical properties, such as electrical conductivity, surface roughness and photoluminescence (PL) of the thin films of an Al-doped $\mathrm{ZnO}$ (AZO-5), are studied.

\section{Experimental}

Thin films of an $\mathrm{Al}_{2} \mathrm{O}_{3}$ (5\%)-doped $\mathrm{ZnO}$ (AZO-5) were prepared by RF magnetron sputtering at $673 \mathrm{~K}$ substrate. Sputtering was carried out at a pressure of $6 \times 10^{-3}$ Torr 
in pure argon gas with RF power $50 \mathrm{~W}$. An amorphous glass plate was used as a supporting substrate for the films. The glass plate deposited with AZO-5 thin films of $900 \mathrm{~nm}$ thickness was cut into pieces of $10 \mathrm{~mm}$ in width and $20 \mathrm{~mm}$ in length. The multiple positive electropulse was applied. We carried out electropulsing treatment (EPT) on the deposited thin films for various periods of time. Operation parameters of pulse frequency and the RMS value of current density were $1.6 \mathrm{kHz}$ and $40 \mathrm{~A} / \mathrm{mm}^{2}$, respectively. The EPT was carried out at room temperature $(300 \mathrm{~K})$.

We did examination of microstructural evolution and the phase decomposition of the non-EPT and EPT AZO-5 thin film specimens by using X-ray diffraction (XRD) and scanning electron microscopy (SEM) techniques. The XRD was performed using an X-ray diffractometer (RIGAKU Smart Lab 9000w) with Ni-filter $\mathrm{Cu} K_{\alpha}$ radiation $(\lambda=0.15418 \mathrm{~nm})$, and scan range was between $2 \theta=20^{\circ}$ and $70^{\circ}$. The scanning speed was $1 \% \mathrm{~min}$. Secondary electron images were collected, using an SEM Jeol-6490. The electrical conductivity, resistivity, carrier concentration and mobility of the AZO-5 thin films were measured at room temperature using a Hall effect measurement system (ECOPIA HMS-5000). Atomic force microscopy (AFM) examination was carried out using a Scanning Probe Bruker Veeco Nanoscope. PL measurement was carried out using a conventional infrared spectrometer.

\section{Results and Discussion}

\subsection{Circular Spinodal Decomposition and Discontinuous Precipitation}

The as-deposited AZO-5 thin films consisted of only a single nanophase shown in Fig. 1a are XRD patterns of the hexagonal structured nanophase. The XRD pattern shows the $2 \theta$ peaks at $31.87^{\circ}, 34.44^{\circ}, 36.31^{\circ}, 47.55^{\circ}, 56.71^{\circ}$, $62.94^{\circ}$ and $68.05^{\circ}$ corresponding to $(10-10)$, (0002), (10-11), (10-12), (11-20) and (10-13) planes, respectively.

Two ways of decomposition of supersaturated phases, i.e., continuous spinodal decomposition, and discontinuous decomposition was observed.

During the early $3 \mathrm{~min}$ of electropulsing, the spinodal decomposition occurred. The zones (named after $Z_{1}$ and $\mathrm{Z}_{2}$ ) formed subsequently, as shown in Fig. 1a.

After $5 \mathrm{~min}$ of electropulsing, both the spinodal decomposition and the discontinuous decomposition developed. Discontinuous precipitates are indicated by white arrows in Fig. 1a. Meanwhile, a part of the zones were broken. Black holes appeared, as black arrows indicated in Fig. 1a.
Upon further electropulsing, the broken zones, i.e., the black holes reduced and amount of discontinuous precipitates decreased after $7 \mathrm{~min}$, as shown in Fig. 1a. Both reverse spinodal decomposition and the discontinuous decomposition occurred.

After $15 \mathrm{~min}$ of electropulsing, the reverse phase decompositions developed, and the $Z_{1}$ and $Z_{2}$ appeared again as round circles in shape. This implied that the inner stress field was then homogenized. Meanwhile, the amount of the discontinuous precipitates reduced, as shown in Fig. 1a. After $20 \mathrm{~min}$ electropulsing, only $Z_{1}$ zone was observed. One circulation of the spinodal decomposition and the discontinuous decomposition was observed within 20 min of electropulsing.

It is generally recognized that the spinodal decomposition started with clustering to form zones, transitional phase and metastable phase, i.e., a continuous decomposition, which might be accompanied with a eutectoid decomposition, i.e., discontinuous decomposition [28-30]. The GP zones formed at the early stage of the supersaturated phases, due to fluctuation of composition of the phases of decomposition. After formation of the GP zones, the transitional phase appeared directionally in needle or rods in shape.

In the present study, the electropulsing-induced circulation of both continuous decomposition, i.e., spinodal decomposition, and the discontinuous decomposition was also observed in the AZO-5 thin films. This was for the first time that the phase decompositions and the microstructural evolution of the AZO-5 thin films were explored to be in a similar way which occurred in the bulk and thin films of alloys [28-30].

The driving force for the phase decompositions in the AZO-5 thin films consists mainly of three parts: $\Delta G=\Delta G_{\text {chem }}+\Delta G_{\text {stress }}+\Delta G_{\text {ep }}$, where $\Delta G_{\text {chem }}$ is the chemical Gibbs free energy, $\Delta G_{\text {stress }}$ the stress inducedGibbs induced from the preferred crystal orientation and $\Delta G_{\text {ep }}$ the electropulsing induced-Gibbs free energy.

$\Delta G_{\text {ep }}$ accelerated the phase transformations in two ways: (a) transformation from the supersaturated state approaching the final state $(\Delta G<0)$, while both parts of $\Delta G_{\text {chem }}$ and $\Delta G_{\text {ep }}$ were applied against the preferred crystal orientation induced- $\Delta G_{\text {stress, }}$, and (b) both the reverse phase decompositions from the final state back to a higher-temperature state, $(\Delta G>0)$. For both the reverse spinodal decomposition and the discontinuous precipitation, $\Delta G_{\mathrm{ep}}$ played as the driving force against the $\Delta G_{\text {chem }}$.

It was reported that the electropulsing accelerated phase transformations of bulk $\mathrm{Zn}-\mathrm{Al}$ alloy and $\mathrm{Mg}-\mathrm{Al}$ alloy by factors of at least 6000 and 3400 times, compared with that in aging processes, respectively $[8,15,16]$. Also, the phase decompositions were considerably fastened in the thin films of both EPT Zn-Al alloys and EPT AZO-2 [24, 25]. 


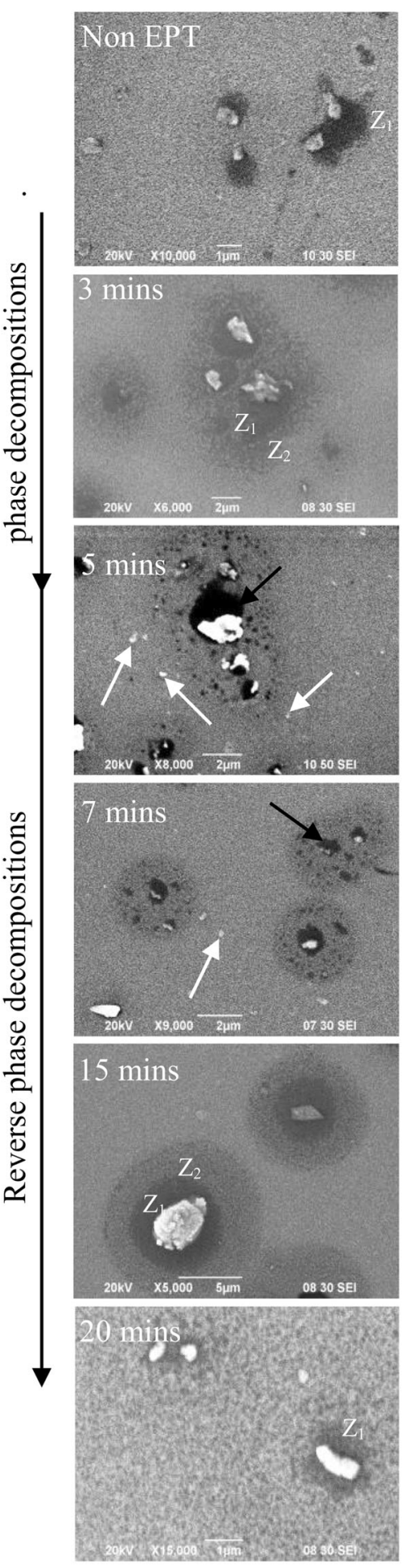

a
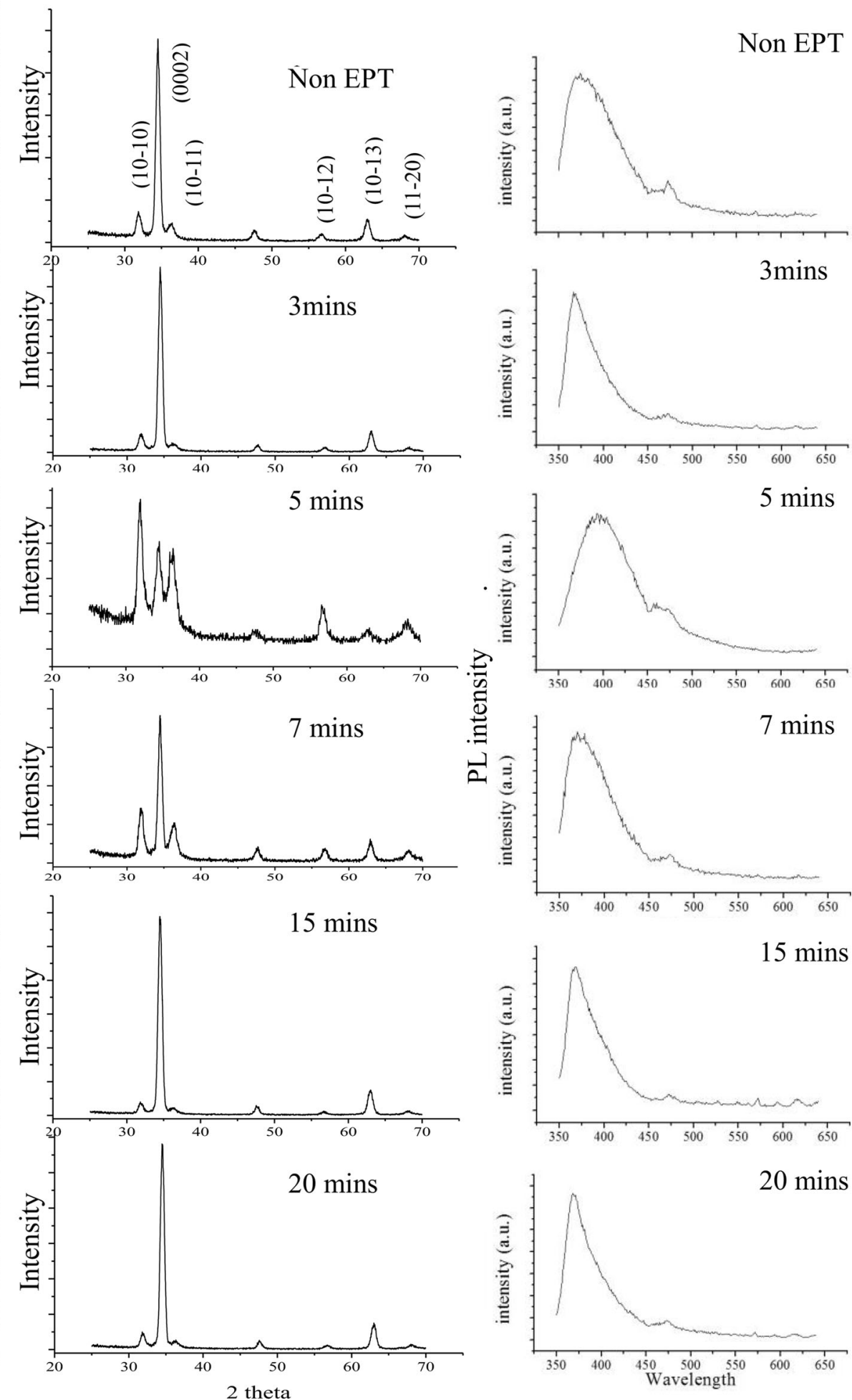

b

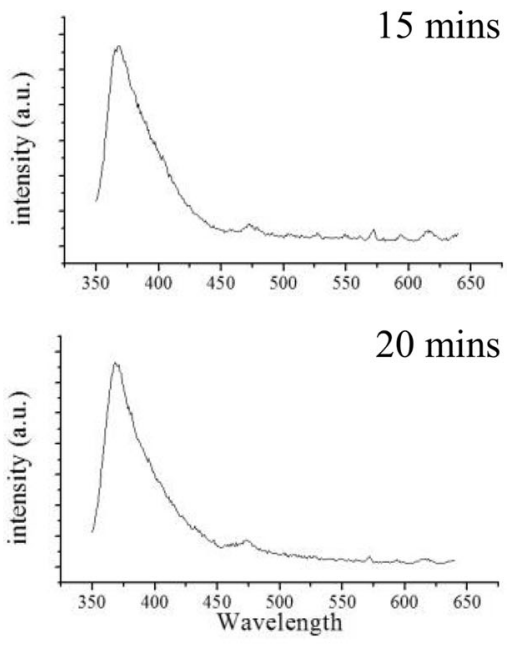

c

Fig. 1 Electropulsing-induced circular spinodal decomposition and discontinuous decomposition in AZO-5 thin films: SEM images a, XRD diffractograms b, PL intensity vs wavelength $\mathbf{c}$ 
In the present study, one circle of phase decompositions and the reverse phase decompositions realized in the EPT AZO-5 thin films within only $20 \mathrm{~min}$ at room temperature.

It was proposed that the $\Delta G_{\mathrm{ep}}$ consisted normally of two

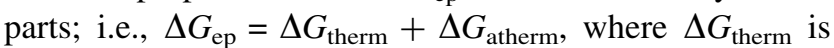
the Gibbs free energy resulting from Joule heat, i.e., the thermal effect. $\Delta G_{\text {atherm }}$ is the Gibbs free energy contributed by the athermal effect. It was estimated that the athermal effect of electropulsing was 319 times stronger than the thermal effect [19]. Thus, during electropulsing, the transfer of energy directly from the electrons to the atoms was much more effective than that in the traditional thermal and thermo-mechanical processes.

Apparently, electropulsing appeared as a powerful and practical process for exploring phase transformations and microstructural changes in the physically stable semiconductor thin films.

\subsection{Prefer Crystal Orientation Changes}

Accompanying the circular spinodal decomposition and the discontinuous precipitation, the preferred crystal orientation changes occurred in the EPT AZO-5 thin films.

As shown in Fig. 1b, strong preferred crystal orientation of the as-deposited AZO-5 thin films appeared at (0002) planes at $2 \theta=34.44^{\circ}$. During the early 5-min EPT, the crystal orientation of the preferred crystal orientation of AZO-5 thin films changed from (0002) planes to (10-10) planes at $2 \theta=31.87^{\circ}$ and $(10-11)$ planes at $2 \theta=36.31^{\circ}$, respectively. Accordingly, electropulsing-induced spinodal decomposition and the discontinuous decomposition occurred.

After $7 \mathrm{~min}$ of electropulsing, the reverse preferred crystal orientation was observed changing from (10-10) planes and (10-11) planes back to (0002) planes, accompanying with recovering the damaged zones. Both the reverse formation of zones and the reverse discontinuous decomposition were observed, as shown in Fig. 1b. After $15 \mathrm{~min}$ of electropulsing, the reverse change in the preferred crystal orientation developed further, accompanying with both of the reverse phase decompositions. After EPT for $20 \mathrm{~min}$, the preferred crystal orientation had considerably changed from (10-10) planes and (10-11) planes back to (0002) planes, as shown in Fig. 1b. Meanwhile, $Z_{2}$ disappeared and the thin film returned to the original non-EPT state, as shown in Fig. 1a.

It was reported that one of the most important characteristics of the as-deposited thin films of the AZO is the strong preferred crystal orientation [26]. Co-relationship between the preferred crystal orientation and the stresses in the thin films was discussed previously [25-27].

In the present study, under electropulsing, the preferred orientation destroyed and changed from the high-stressed
(0002) planes of the as-deposited state to (10-10) planes and (10-11) planes, while the stress reduced to minimum in the EPT AZO-5 thin films. Meanwhile, formation of zones and the discontinuous precipitation occurred. Upon further electropulsing, the reverse crystal orientation was observed. Electron wind appeared as an external stress, forcing the crystal orientation away from the (10-10) planes and (10-11) planes back to the high-stressed (or either external stressed or a high-temperature state) (0002) planes, accompanying the reverse phase transformations. It is clearly seen that the preferred crystal orientation was closely related with stresses in the AZO-5 thin films.

The above-mentioned preferred crystal orientation changed from the high-stressed state to the low-stressed state and the reverse were observed in the thin films of both the $\mathrm{Zn}-\mathrm{Al}$ alloys, the $\left(\mathrm{Bi}_{0.25} \mathrm{Sb}_{0.75}\right)_{2} \mathrm{Te}_{3}$ and $\mathrm{AZO}-2$ semiconductor [24-26].

\subsection{Effect on Electrical Conductivity}

The electrical conductivity $(\sigma)$, electric resistivity $\left(\rho^{-1}\right)$, large carrier concentration $(n)$ and the Hall mobility $(\mu)$ were plotted against various periods of electropulsing $(0,3$, 5, 7, 15 and $20 \mathrm{~min}$ ), as shown in Fig. 2.

The electrical conductivity is related with mobility $(\mu)$ and carrier concentration $(n): \sigma=\rho^{-1}=n e \mu$. With adequate balance between carrier concentration and the mobility, the electrical conductivity increased to a maximum. Effect of the electropulsing-induced phase decomposition, and structural evolution on the electrical conductivity $(\sigma)$ became complicated.

At early stage of electropulsing, the strong preferred crystal orientation was destroyed [24], and the dislocation density increased [25-27]. Meanwhile, the residue stress in the as-deposited AZO-5 thin films decreased. The interaction between electrons and the stress field became weak. The electrical conductivity increased. Because of the spinodal decomposition, i.e., the formation of zones, the mobility increased and the carrier concentration decreased, which resulted in the decrease in the electrical conductivity. As a result, the electrical conductivity increased eventually after $3 \mathrm{~min}$ of electropulsing.

The electropulsing-induced phase transformations influenced the dislocation dynamics. After 5 min of electropulsing, the discontinuous precipitation developed. The electropulsing-induced discontinuous precipitates caused dislocation pinning, which increased density of dislocation [25-27]. Both the carrier concentration and vacancies increased, which favored increasing electrical conductivity [25-27]. However, because of the damage of the zones which resulted in the decrease in both densities of carrier concentration and the mobility greatly, the electrical conductivity tremendously decreased. Meanwhile, the 


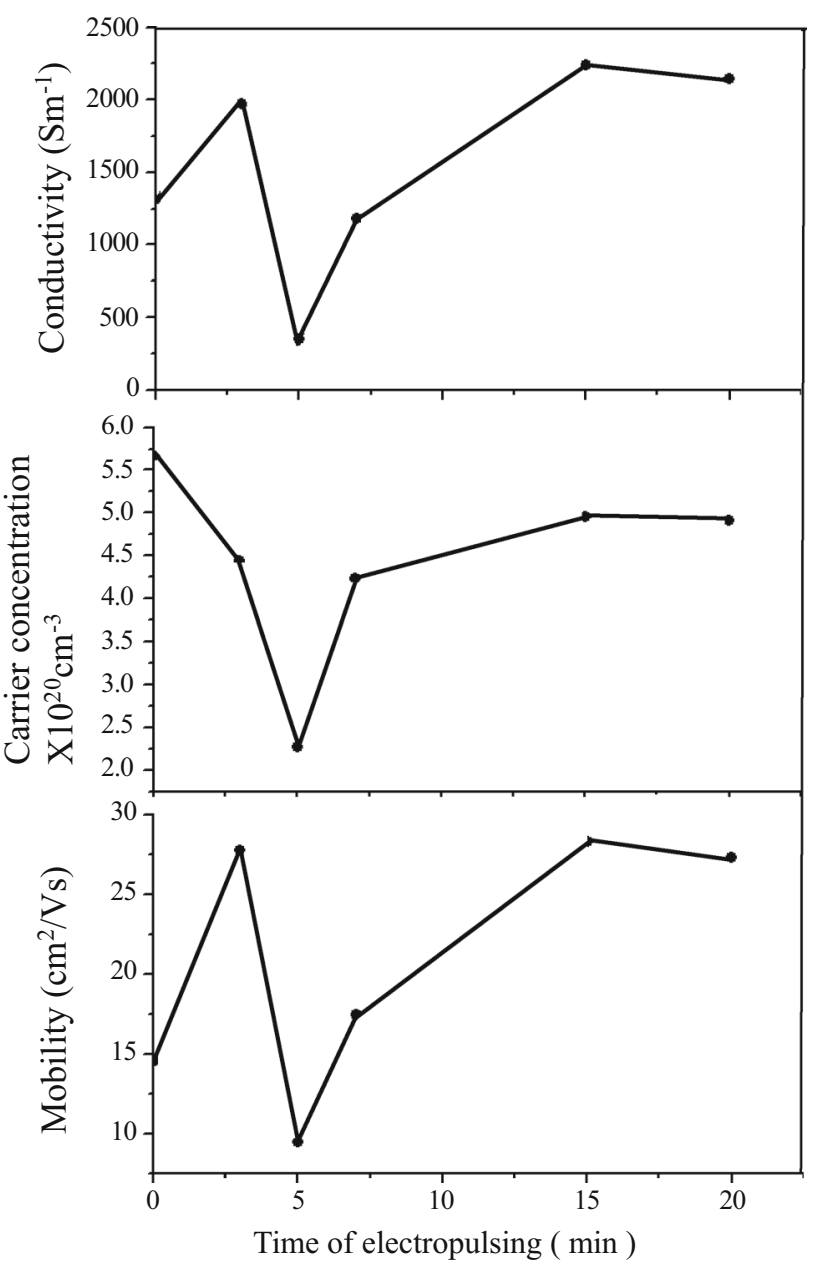

Fig. 2 Electrical conductivity, electrical resistivity, carrier concentration and mobility of AZO-5 thin films after various periods of electropulsing

preferred crystal orientation changed from (2000) planes to (10-10) planes and (10-11) planes.

Upon further electropulsing, because the damaged zones recovered, which played a dominant role in the increase in both densities of carrier concentration and the mobility, the electrical conductivity increased greatly. The reverse spinodal decomposition decreased the mobility and the reverse discontinuous precipitation reduced the carrier concentration that decreased the electrical conductivity. As a consequence, the electrical conductivity considerably increased. After $15 \mathrm{~min}$ of electropulsing, the electrical conductivity enhanced to $2.243,13 \%$ higher than that of the non-EPT specimen. The preferred crystal orientation changed accordingly from (10-10) planes and (10-11) planes back to (2000) planes.

It can be seen that the discontinuous precipitation and the preferred crystal orientations at (10-10) planes and (10-11) planes favored electrical conductivity of the AZO5 thin film. Inappropriate electropulsing might damage the zones, which resulted in the tremendous decrease in electrical conductivity.

\subsection{Roughness of the AZO Thin Films}

The surface roughness is one of the most important properties of the thin films of AZO, as far as the application is concerned. The roughness was presented by the root mean square (RMS) value $R_{\mathrm{q}}$. Shown in Figs. 3 and 4 are AFM images and the RMS values of AZO-5 thin films after electropulsing for $0,3,5,7,15$ and $20 \mathrm{~min}$. The RMS value $R_{\mathrm{q}}$ of the as-deposited thin film was 20.8. After 3 min of EPT, the supersaturated solid phase had started to cluster and the zones $Z_{1}$ and $Z_{2}$ formed, as shown in Fig. 1a, and the RMS value decreased to 9.70 . After $5 \mathrm{~min}$ of electropulsing, the zones were well developed and parts of the zones were damaged. As a result, the roughness of the thin films reduced considerably to $R_{\mathrm{q}}=2.78$.

Upon further electropulsing, the RMS values increased because the broken zones were recovered and the reverse spinodal decomposition occurred, as shown in Figs. 1a, d, 3 and 4. This implied that the spinodal decomposition, i.e., formation of the zones, favored reducing roughness of the thin films.

\subsection{Photoluminescence (PL)}

The PL bank gap energy is expressed as $E_{\mathrm{g}}=h c / \lambda$, where $h$ Plank's constant, $c$ light speed $3 \times 10^{8} \mathrm{~ms}^{-1}, \lambda$ wavelength. $E_{\mathrm{g}}$ (band gap energy) of $\mathrm{ZnO}$ is $3.37 \mathrm{eV}$, and then $\lambda$ is calculated to be $380 \mathrm{~nm}$ (UV). The PL intensity stands for the PL band gap energy. The PL intensity versus wavelength and PL peak wavelength position of the AZO-5 thin films after various periods of electropulsing are shown in Figs. 1c and 5, respectively.

Peak width implies structure nature. Narrow peak means the single-phase structure, and wider peak, a multiphase structure. During the early $5 \mathrm{~min}$ of electropulsing, phase decompositions occurred and the zones and discontinuous precipitates were observed. The multiphase structure resulted in widening of the PL peak. The peak wavelength shifted to higher-value direction, so-called the red shifting, as shown in Figs. 1c and 5.

Upon further electropulsing, the PL wavelength peak narrowed and shifted back to lower-value direction, i.e., the blue shifting, as shown in Figs. 1c and 5. This means that the multiphase structure gradually changed back to the single-phase structure, i.e., the reverse decompositions occurred.

The above-mentioned circular phase decompositions were agreed with that from examination of the XRD and SEM of the thin films of AZO-5. 


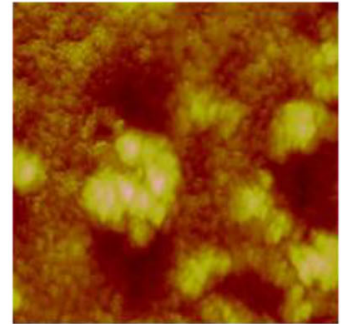

Scan Area: 5 um $\times 5$ um Rq (r.m.s.): $20.7 \mathrm{~nm}$

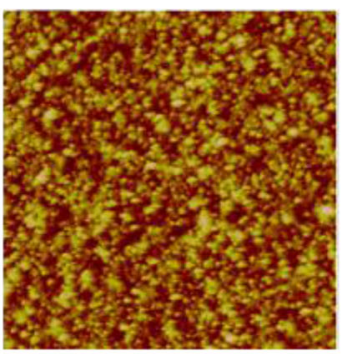

Scan Area: 5 um $\times 5$ um

Rq (r.m.s.): $9.70 \mathrm{~nm}$

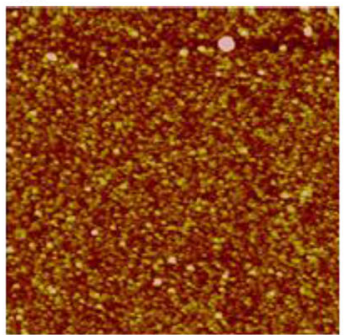

Scan Area: 5 um $\times 5$ um

Rq (r.m.s.): $2.78 \mathrm{~nm}$

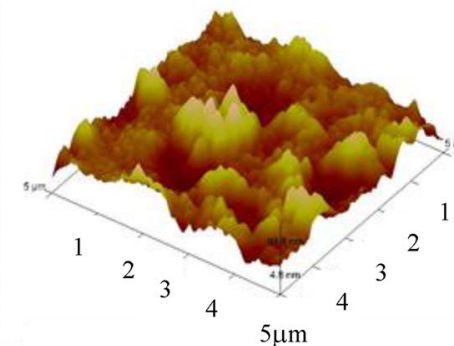

$0 \min$

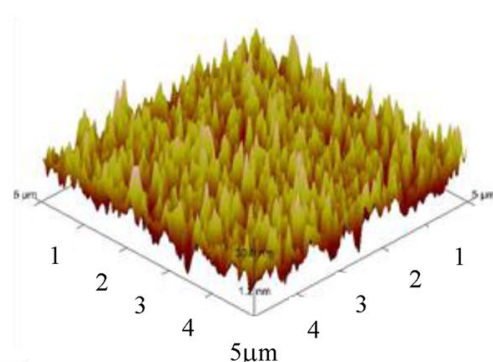

$3 \min$

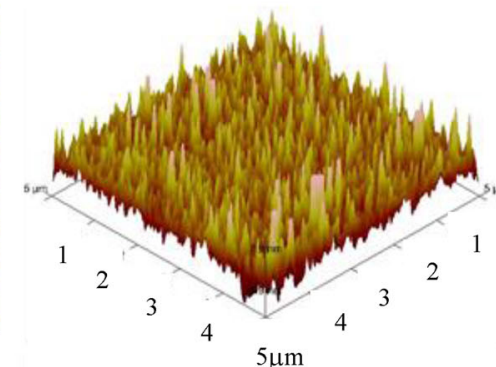

$5 \mathrm{~min}$

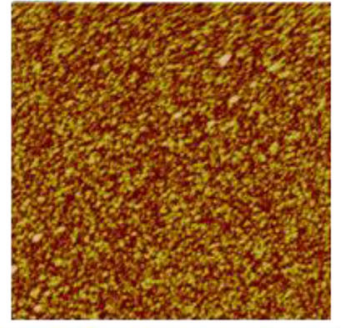

Scan Area: 5 um x 5 um

Rq (r.m.s.): $8.73 \mathrm{~nm}$

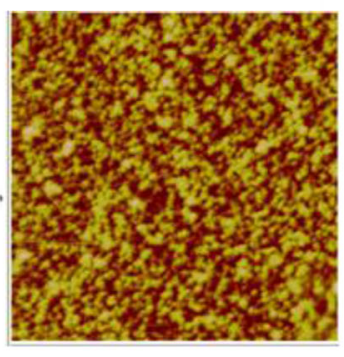

Scan Area: 5 um $\times 5$ um

Rq (r.m.s.): 16.5 nm

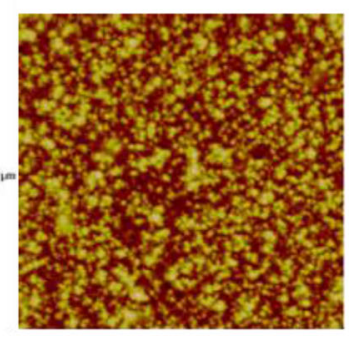

Scan Area: 5 um $\times 5$ um

Rq (r.m.s.): 18.7 nm

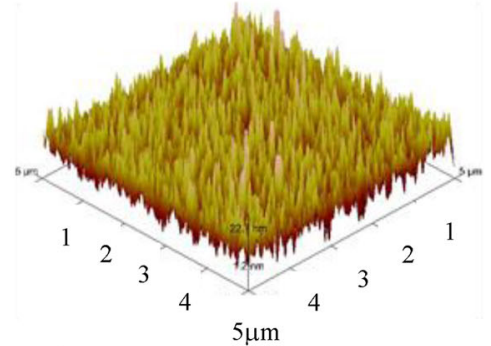

$7 \min$
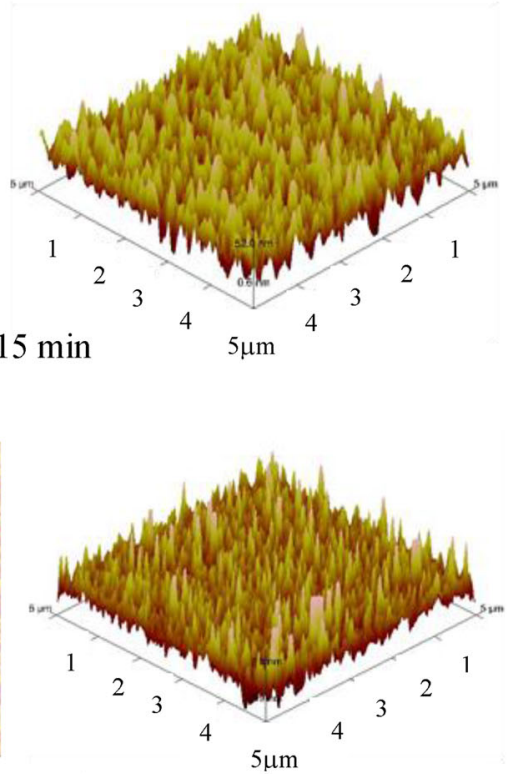

$20 \min$

Fig. 3 AFM images of AZO-5 thin films after various periods of electropulsing

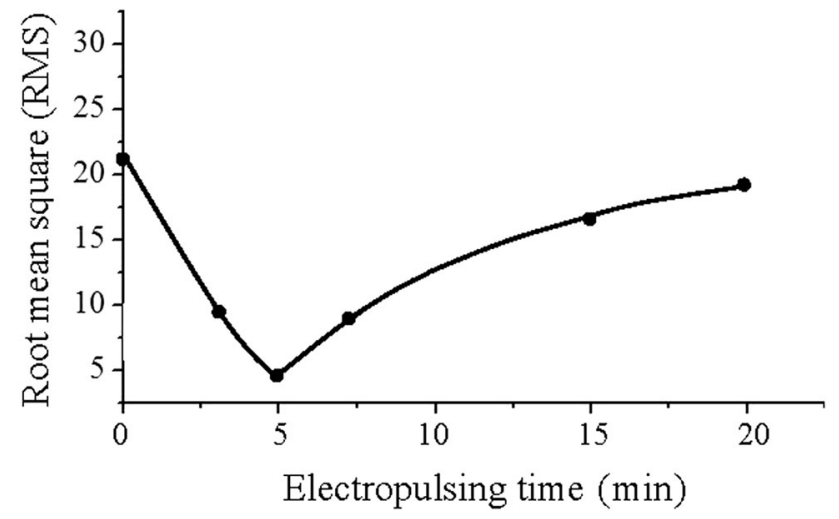

Fig. 4 Root mean square (RMS) values of AZO-5 thin films after various periods of electropulsing $(0,3,5,9,15$ and $20 \mathrm{~min})$

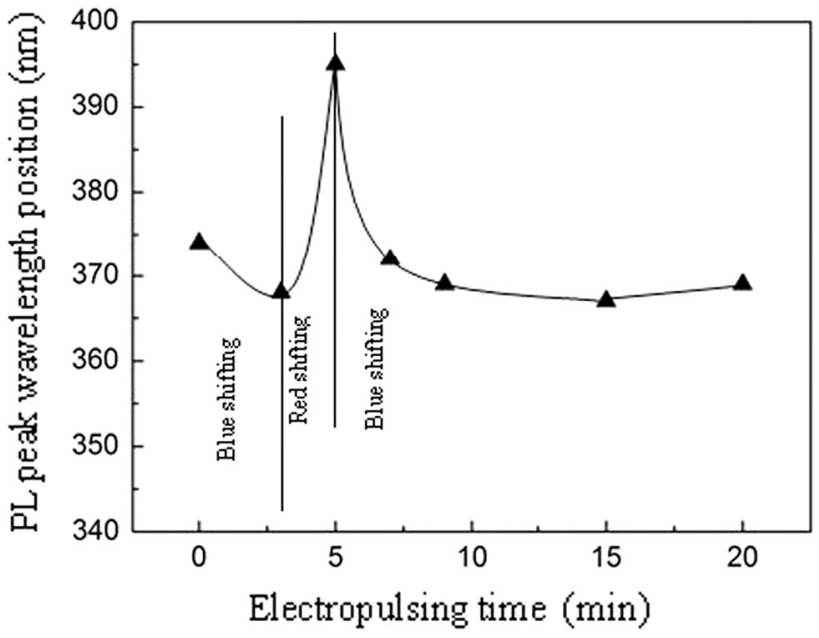

Fig. 5 PL peak wavelength position vs electropulsing time of AZO-5 thin films, showing the twice blue shifting 
It was interesting to notice that within the early $3 \mathrm{~min}$ of electropulsing, a wider PL wavelength peak of the as-deposited thin films had narrowed and shifted to low-value direction, i.e., the blue shifting, due to the destroying of the high-stressed orientation at (2000) at the early stage of electropulsing [25], as shown in Figs. 1c and 5.

Occurrence of the twice blue shifting (Fig. 5) implied that the phase decompositions played a dominant role in changing of the PL peak wavelength value, i.e., of both blue shifting and the red shifting. The destroying of the heterogeneous microstructure and the high-stressed orientation at (0002) planes favored reducing PL peak wavelength value, i.e., the first blue shifting, at the early stage of electropulsing.

\section{Conclusions}

1. Electropulsing-induced phase decompositions started with formation of zones, which was accompanied by a discontinuous precipitation in the AZO-5 thin films.

2. The electropulsing induced the circular continuous decomposition, i.e., spinodal decomposition, and the discontinuous decomposition was observed in the AZO-5 thin films.

3. The electropulsing-induced discontinuous precipitation and the preferred crystal orientations at (10-10) planes and $(10-11)$ planes favored the electrical conductivity of the AZO-5 thin films. Inappropriate electropulsing might damage zones, which resulted in the decrease in electrical conductivity tremendously.

4. The circular phase decompositions played an important role in changing of the PL peak wavelength value and the peak width in the EPT thin films of AZO-5. The destroying of the heterogeneous microstructure and the high-stressed orientation favored reducing the PL wavelength peak width and the peak value at the early stage of electropulsing.

5. Spinodal decomposition, i.e., the formation of the zones, favored roughness of the EPT thin films of the AZO-5.

\section{References}

[1] S. Parthiban, E. Elangovan, K. Ramamurthi, R. Martins, E. Fortunato, Solar energy mater. Solar Cells 94, 406 (2010)

[2] H. Kelmer, Nat. Phonton. 6(808), 816 (2012)

[3] E. Fortunato, Adv. Mater. 24, 2945 (2012)

[4] L. Castaneda, Mater. Sci. Appl. 2, 1233 (2011)

[5] P.A. Fluga, V. Sittingera, F. Ruskea, B. Szyszkaa, G. Dittmarb, Thin Solid Films 455-456, 201 (2004)

[6] M. Hiramatsu, I. Kmaeda, N. Horio, M. Nawata, J. Vac. Sci. Technol. A 16, 669 (1998)

[7] Y.H. Zhu, S. To, W.B. Lee, X.M. Liu, Y.B. Jiang, G.Y. Tang, Mater. Sci. Eng. A 501, 125 (2009)

[8] Y.H. Zhu, S. To, W.B. Lee, X.M. Liu, Y.B. Jiang, G.Y. Tang, J. Mater. Res. 24, 2661 (2009)

[9] Y.H. Zhu, S. To, X.M. Liu, Metall. Mater. Trans. A 42, 1933 (2011)

[10] Y.H. Zhu, S. To, X.M. Liu, Mater. Trans. JIM 53, 1363 (2012)

[11] Y.H. Zhu, S. To, X.M. Liu, J. Microsc. 242, 62 (2011)

[12] Y.H. Zhu, S. To, X.M. Liu, G.L. Hu, Q. Xu, J. Mater. Res. 26 , $1696(2011)$

[13] Y.B. Jiang, G.Y. Tang, C.H. Shek, Y.H. Zhu, Z.H. Xu, ACTA 57, 4797 (2009)

[14] Y.B. Jiang, G.Y. Tang, C.H. Shek, Y.H. Zhu, L. Guan, S.N. Wang, Z.H. Xu, J. Mater. Res. 24, 1810 (2009)

[15] Y.B. Jiang, G.Y. Tang, C.H. Shek, Y.H. Zhu, Appl. Phys. A 97, 607 (2009)

[16] D. Zhang, S. To, Y.H. Zhu, H. Wang, G.Y. Tang, Int. J. Mater. Res. (Zeit Metallkunde) 21, 1205 (2012)

[17] D. Zhang, S. To, Y.H. Zhu, H. Wang, G.Y. Tang, Metall. Mater. Trans. A 43, 1341 (2012)

[18] D. Zhang, S. To, Y.H. Zhu, H. Wang, G.Y. Tang, J. Surf. Eng. Mater. Adv. 6, 106 (2016)

[19] G.L. Hu, G.Y. Tan, Y.H. Zhu, C.H. Shek, Metall. Mater. Trans. A 42, 3484 (2011)

[20] G.L. Hu, Y.H. Zhu, C.H. Shek, G.Y. Tang, J. Mater. Res. 26, 917 (2011)

[21] Z.H. Xu, G.Y. Tang, S.Q. Tian, J.C. He, Mater. Sci. Eng. A 424, 300 (2006)

[22] V.V. Stolyarov, Mater. Sci. Eng., A 503, 18 (2009)

[23] V.E. Gromov, Y.F. Ivanov, E.V. Kozlov, E.Y. Suchkova, S.V. Konovaloc, V.A. Rybyyanets, in Y.V. Baranov, V.E. Gromov G.Y. Tang (ed.), Proceedings (2009), pp. 209-224

[24] Y.H. Zhu, S.Q. Jiang, D.D. Zhao, G.J. Cheng, H.W. Zhang, W.E. Lai, Appl. Phys. A 111, 1241 (2013)

[25] Y.H. Zhu, J. Jiang, Y.K. Xiao, C.M. Luk, W.E. Lai, Scr. Mater. 69, 219 (2013)

[26] Y.H. Zhu, W.E. Lai, J. Surf. Eng. Mater. Adv. Technol. 6, 106 (2016)

[27] C.N. Liao, K.M. Liou, H.S. Chu, Appl. Phys. Lett. 93, 042103 (2008)

[28] Y.H. Zhu, Mater. Trans. JIM 45, 3083 (2004)

[29] Y.H. Zhu, C.Y. Chung, W.B. Lee, Mater. Sci. Eng. A 374, 145 (2004)

[30] Y.H. Zhu, W.B. Lee, S. To, J. Mater. Sci. Technol. 20, 687 (2004) 